\title{
Probing the local response of a two-dimensional liquid foam ${ }^{\star, \star \star}$
}

\author{
Leevi Viitanen ${ }^{1, a}$, Juha Koivisto ${ }^{1}$, Antti Puisto ${ }^{1}$, Mikko Alava ${ }^{1}$, and Stephane Santucci ${ }^{2,3}$ \\ 1 Aalto University, Department of Applied Physics, PO Box 11100, 00076 Aalto, Finland \\ ${ }^{2}$ Université de Lyon, ENSL, UCBL, CNRS, Laboratoire de Physique, Lyon, France \\ ${ }^{3}$ Lavrentyev Institute of Hydrodynamics, Novosibirsk, Russia
}

Received 19 June 2018 / Received in final form 17 December 2018

Published online 13 February 2019

(C) The Author(s) 2019. This article is published with open access at Springerlink.com

\begin{abstract}
Aqueous foams are viscoelastic yield stress fluids. Due to their complex rheology, foam flow around an obstacle embedded in a 2D Hele-Shaw cell has been widely studied. Typically, in such geometry in the moving flow reference frame the flow field of viscoelastic fluids exhibit a quadrupolar structure characterized by a negative wake. Here, we introduce a measuring geometry, new in this context, whereby instead of flowing the foam around the obstacle, we move the obstacle as an intruder inside the foam. The proposed setup makes it possible to independently control the driving velocity and the liquid foam properties, such as the gas fraction and polydispersity. We show that the liquid foam velocity field around the intruder is similar to the one observed in viscoelastic fluids, in particular the emergence of a negative wake, e.g. a velocity overshoot downstream side of the obstacle. However, surprisingly, the intensity of this velocity overshoot decreases with the number of intruder passes, probably related to the evolution of the local disordered structure of the liquid foam.
\end{abstract}

\section{Introduction}

Aqueous foams are made of a mixture of gas, liquid and surfactants, where the gas is phase separated into bubbles. Their rheological character varies depending on their gas volume fraction $\phi[1-3]$. A critical volume fraction, which controls the qualitative rheological behavior of foams depends on the system dimensionality with the typical reported values of $\phi_{c}=0.64(3 \mathrm{D})$ and $\phi_{c}=0.82$ (2D). However, $\phi_{c}$ depends also on the bubble polydispersity and can reach values as large as $\phi_{c}=0.87$ (in 2D) [4]. At low $\phi<\phi_{c}$, at the bubbly liquid regime, the bubbles are not forced to touch one another, and the rheological characteristics of the foam falls into the category of linear, or Newtonian, fluids [5]. Above $\phi_{c}$ the bubbles lose their spherical shape and form a disordered structure in a metastable state at a local surface energy minimum $[6,7]$. The force restoring the system to the local energy minimum is due to the surface tension between the two phases [8]. Thus, for foams with $\phi>\phi_{c}$ this force sets a threshold for the external driving, called the yield stress [9]. At external driving below the yield stress, the

\footnotetext{
*Contribution to the Topical Issue "Complex Systems Science meets Matter and Materials", edited by Stefano Zapperi.

${ }^{\star}$ Supplementary material in the form of one mp4 file available from the Journal web page at

https://doi.org/10.1140/epjb/e2019-90402-x

${ }^{a}$ e-mail: leevi.viitanen@aalto.fi
}

bubble configuration remains unchanged. In this regime, under the conditions of slow forcing the shear response of foams is described by linear elasticity. However, the linear elastic regime of foams, especially those close to the wet limit, can be very narrow [10]. At external stresses above the yield stress, as oppose to solid-like breaking, a foam begins to flow. This occurs as a consequence of a series of bubble configuration changes via local topological neighbor swap events, called T1's [11,12]. Thus, aqueous foams lie in between fluids and solids as they possess the properties of both $[6,13,14]$. This makes them an ideal playground for studying the interplay of elasticity and plasticity.

The phenomenology found in these systems is even richer. The dynamics of foams also depend on their polydispersity: monodisperse foams form ordered structures, while sufficiently polydisperse ones remain in an amorphous state. The dynamics of these two systems are fundamentally very different $[15,16]$. In ordered foams the flow occurs via simultaneous neighbor swaps at the interfaces of large ordered domains of bubbles [15]. However, in disordered foams the dynamics is more complicated as it is based on discrete and irregular events of neighbor swaps, which occur already at modest deformations [10,16]. Therefore, the local response of amorphous foams is justifiably expected to be highly nontrivial, making them an ideal material for the present research.

Foam flow past a circular obstacle in a two-dimensional setup is a very commonly, and in-depth studied scenario [17-21]. These studies find a breaking of the 
fore-aft flow symmetry, and a velocity overshoot after the obstacle. The flow field is similar to the negative wake [22] behind a moving object in complex fluid (as oppose to the undershoot typically observed in the wake of an object moving in Newtonian fluid), which results in a quadrupolar velocity field that forms around the obstacle. Earlier studies involve an extensive set of parameters such as the flow rate, bubble area, fluid fraction, bulk viscosity, obstacle size and boundary conditions (at the obstacle) [19]. In addition, the influence of obstacle shape and height was only recently considered [23]. Based on a detailed analysis utilizing different levels of continuum models, the quadrupolar flow field was attributed to the elastoviscoplastic (EVP) properties of the foam [24]. This is in line with the observations that the negative wake appears in other pseudoplastic viscoelastic fluids as well. In these materials, it occurs either when such fluid confronts an obstacle or is deformed with an intruder (such as a bubble, a sedimenting particle, or a sphere). What distinguishes foam from the other viscoelastic fluids is its larger size of the building blocks (compare bubbles with a typical size of a polymer).

Majority of studies reviewed so far focus on the flowing foam and fixed obstacle. However, a few experiments where intruder, or pair of intruders, are driven through the foam by gravity have been reported $[25,26]$. There, an attraction between subsequently dropped intruders was observed, indicating that the foam structure relaxes due to the motion of the first intruder.

In this article, we study the use of an intruder setup to measure foam dynamics in a two dimensional HeleShaw cell. In the more usual setup, foam is driven past an obstacle by generating new bubbles on the fly, which push the foam forward. This introduces a coupling between the bubble size distribution, the gas volume fraction and the driving velocity. The proposed setup decouples these properties allowing to set the driving velocity without influencing the bubble size distribution nor the gas fraction. This gives a very attractive possibility to study the local rheology of liquid foam as a function of the bubble size distribution properties at different driving rates, difficult to achieve on a flowing foam scenario. However, the drawback is that the system is exposed to coarsening and foam drying [27], which limit the maximum experiment duration [28]. Here, the duration of the experiments of $24 \mathrm{~min}$ is chosen so that foam aging by coarsening is negligible, however, we do observe a few occasional film breaks during the experiment caused by the intruder. Furthermore, as we demonstrate in this article, the experiment also exposes a dependence of the velocity field on the number of intruder passes. This demonstrates that the viscoelastoplastic response of the foam is highly dependent of the topological frustration due to local bubble geometry.

The rest of the article is organized as follows. In Section 2, we introduce our materials and methods including the detailed experimental geometry. In Section 3, we measure the displacement fields and analyze the foam flow around the intruder. The paper finishes with the conclusions and future prospects. (a)

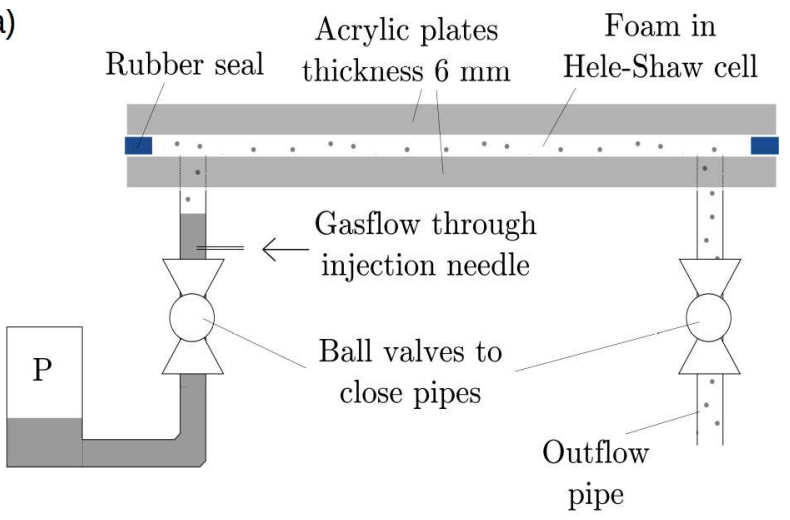

(b) $560 \mathrm{~mm}$



Fig. 1. Schematic diagram (a) displays a schematic representation of the experimental geometry. Diagram (b) shows the top view of the layout including the dimensions of the Hele-Shaw cell. The shaded area indicates the cell. The inlet and outlet holes are closed during the measurements.

\section{Methods}

The experiments are performed in a quasi-twodimensional geometry where the sample foam consists of a single layer of bubbles. The bubble monolayer is confined between two parallel plates. A schematic figure of the device is shown in Figure 1a. Soap-water solution is prepared using Fairy dish washing liquid, which contains various surfactants such as sodium dodecyl sulfate, sodium laureth sulfate and lauramine oxide. The amount of Fairy in the solution is $\sim 1.1$ vol.\%. The solution is contained in a bottle which has the pressure $P>P_{a t m}$. The overpressure pushes the liquid out from the bottle through a pipe leading to the Hele-Shaw cell. The bubbles are formed by injecting butane in the liquid through an injection needle. Foam is fed to the system through a single inlet hole. The layout of the cell is shown in Figure 1b. The cell dimensions are $560 \times 480 \mathrm{~mm}^{2}$ and the gap width of $3 \mathrm{~mm}$. All the inlet and outlet pipes are equipped with a valve allowing to control the flow through each of them separately.

The top and bottom plates are made of transparent acrylic plastic to allow performing the measurements using an optical camera. The camera used in the measurements is a Canon EOS M3 digital camera equipped with Canon EF $75-300 \mathrm{~mm}$ lens that records the video with 

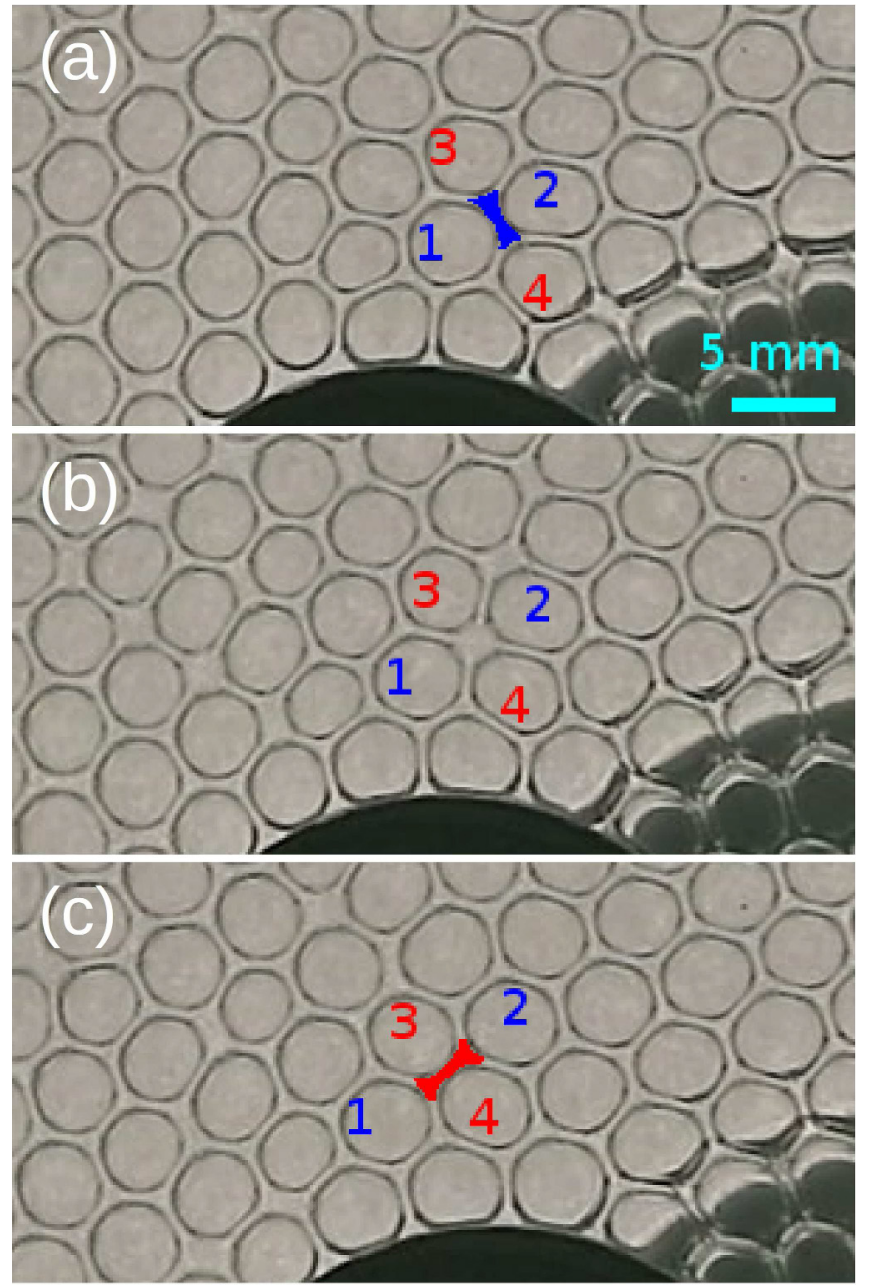

Fig. 2. A Series of raw images near the obstacle showing a single topological event. The length scale to all three images is indicated with the cyan bar. (a) Bubbles 1 and 2 are neighbors at $t=0 \mathrm{~s}$. (b) The flow geometry forces bubbles 1 and 2 to move away from each other reducing the film between them into a fourfold node, $t=0.4 \mathrm{~s}$. (c) A T1 event takes place and bubbles 3 and 4 become neighbors, $t=0.9 \mathrm{~s}$.

$1920 \times 1080$ resolution with $25 \mathrm{~Hz}$. The video is then converted to still images with 0.1 second time difference for simplicity. Figure 2 shows a series of raw images near the intruder highlighting a T1 event next to the intruder.

The experiment works as follows. First, the cell is filled with foam by pumping water/surfactant mixture to the inlet pipe, where the gas is mixed to the solution through a thin needle. Meanwhile, the excess gas is allowed to exit the cell via the outlet pipes carefully ensuring the foam completely fills the entire cell. Finally, the foam is forced still by closing the valves of the inlet and outlet pipes. The experiment is started 30 seconds after the foam is generated and the total duration of the experiment is around 24 minutes. The resulting foam consists of bubbles with radius $r=2.0 \mathrm{~mm}$ and polydispersity measured by the standard deviation of $\Delta r=0.3 \mathrm{~mm}$, see Figure 2 . The gas fraction estimated from the gray scale images is $\phi=0.80 \pm 0.03$, close to the bubbly liquid limit. However,

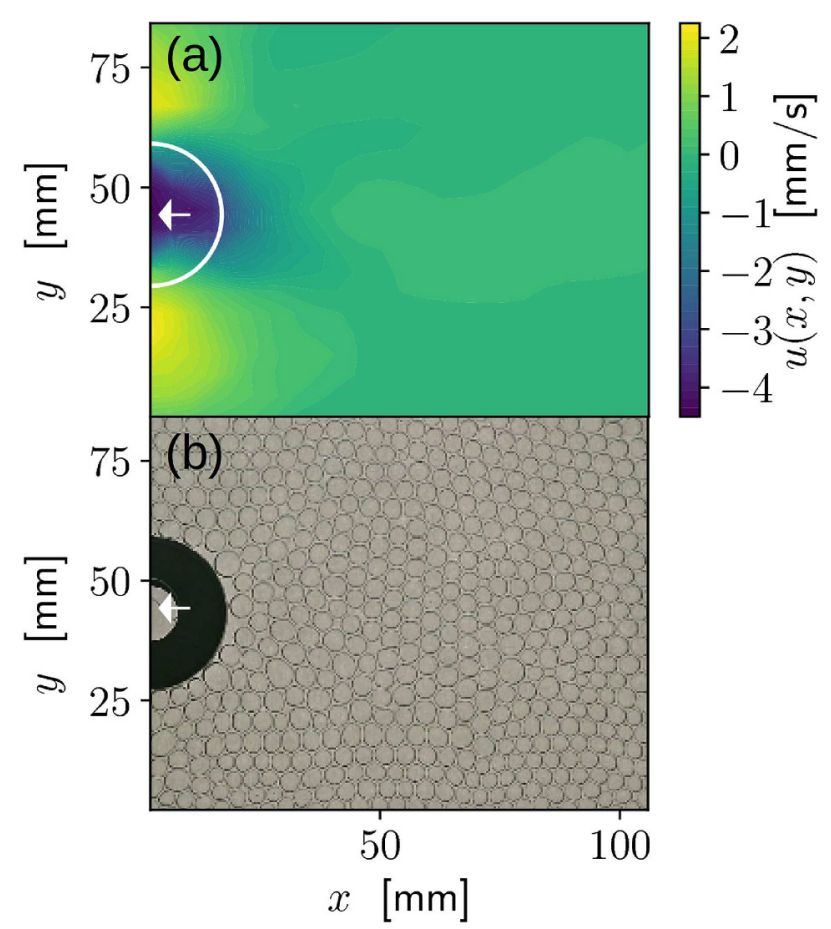

Fig. 3. Foam velocity field behind the intruder. The top panel shows the magnitude of the velocity component in the $x$-direction averaged over time. The semicircle on the left shows the location of the intruder. In the lower panel, we include the image of the sample at the positions corresponding to the velocity field.

the bubbles are not circular as seen in Figure 2 indicating that we are above $\phi_{c}$. The volume fraction is determined by binarizing the image to gas and liquid by hand and calculating the ratio from a single sample image.

The intruder is a round metal disk with the diameter of $29.8 \mathrm{~mm}$ and thickness of $2.6 \mathrm{~mm}$, partially filling the $3 \mathrm{~mm}$ thick cell gap. It is dragged through the foam using a magnet outside the cell. The driving velocity is set to $u_{d}=4.3 \mathrm{~mm} / \mathrm{s}$.

Digital particle image velocimetry technique is used to determine the velocity fields [29]. To do this, the images are divided into subsequent pairs with $\Delta t=100 \mathrm{~ms}$ interval and the local displacements in the sample are calculated between each image pair. The spatial scale is determined by PIV with the tile size of $4.32 \times 4.32 \mathrm{~mm}^{2}$. Velocity fields are then computed from the displacement fields with respect to the intruder center, and the mean velocity field is calculated by averaging over a sufficiently large amount of samples. The time of single pass and occasional stick slip determine an upper limit for the number of samples used for averaging. The resulting velocity fields are averages of $80-120$ samples, which corresponds to averaging over periods of $16-24 \mathrm{~s}$.

\section{Results}

As mentioned earlier, in a typical Hele-Shaw cell setup the driving velocity of the flowing foam is dependent on the fluid and gas pressures that are coupled. These, on the 

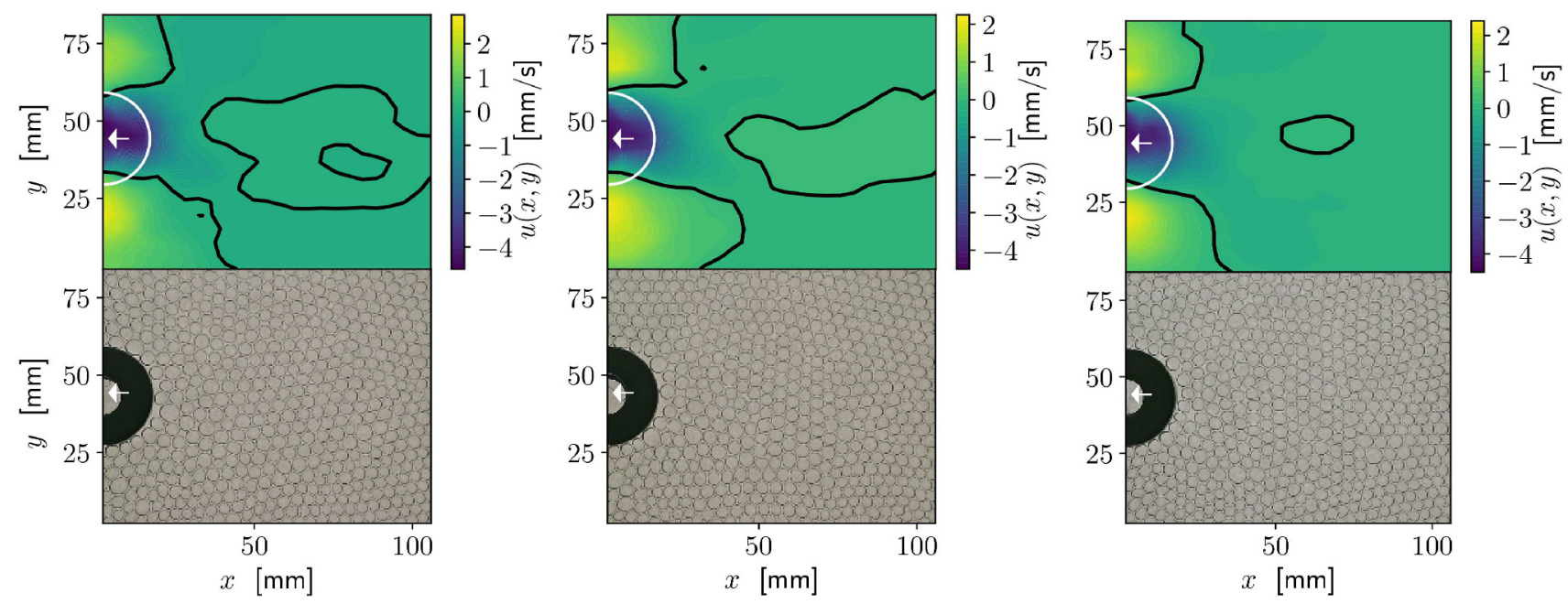

Fig. 4. The $x$-component of the velocity field $u(x, y)$ at different times during the course of the experiment. The velocity contour $u=0 \mathrm{~mm} / \mathrm{s}$ is highlighted in each image. The left panel shows the velocity averaged over frames between 116 and $132 \mathrm{~s}$, in the middle panel the average is taken between 208 and $224 \mathrm{~s}$, and on the right panel the average is over 868 and $892 \mathrm{~s}$. Comparison between the panels show that the negative wake becomes smaller as the time advances. From each time range, an example image shows the sample foam behind the obstacle.

other hand, set the bubble size and polydispersity of the foam and its gas volume fraction. In the intruder setup presented here, the gas volume fraction of the foam can be tuned as there is no liquid and gas flow during the experiment; The setup allows to set the driving velocity and the foam properties independently. However, in the typical setup the foam is generated 'on the fly', thus, the foam is pristine whenever it hits the obstacle. Here, on the other hand, the foam reorganizes after each intruder pass, which will interfere the foams internal stress field.

We show the velocity field measured in a typical experiment with a single pass of the intruder in Figure 3. Specifically we plot the velocity field $x$-component, $u(x, y)$, behind the intruder, time averaged over the whole experiment in Figure 3a, and the corresponding sample image in Figure $3 \mathrm{~b}$. The plot shows the typical characteristics of a quadrupolar velocity field behind the intruder. It consists of (i) the decrease of $u(x, y)$ at strips going $\pm 45^{\circ}$ angle behind the intruder and (ii) a maximum at the distance of roughly $45 \mathrm{~mm}$ measured from the intruder center. This maximum behind the intruder is known as the negative wake and it is found to form in various non-Newtonian fluids with elastic properties such as polymer solutions [22]. The flow field structure is remarkably similar to the one observed in a liquid foam forced to flow around an obstacle $[19,20,23]$ despite the fact that here only a small portion of the bubbles are involved in the process.

The proposed setup naturally rises the question, how the liquid foam structure and dynamics evolve when an intruder is repeatedly driven through it. We studied the evolution of the sample by observing the wake behind the obstacle on different times. A measurement took roughly 24 minutes, during which the obstacle was dragged back and forth in cycles of 95 seconds. For the analysis we choose three periods when the obstacle moves smoothly and the direction of the movement is the same. For these sets we calculate the average flow field behind the obstacle. We plot the data in Figure 4 in chronological order from left to right. The negative wake becomes smaller as the number of passes increases (and time advances). The quadrupolar shape of flow field remains, but its intensity becomes weaker at each pass. Observing the still images below the velocity maps, it proves to be very difficult to pin-point a reason for such behavior. The individual bubbles naturally change during each pass, however the structure is not seemingly more disordered or ordered as the number of passes increases. This is perfectly in line with the well known fact that the bubble size distribution is the main factor determining the structural disorder in foams [30].

Further details to the situation are provided by the velocity $u(x, y)$ profile behind the intruder along the centerline. In Figure 5, we plot the centerline averaged velocity over a single pass on the 3rd pass, 5th pass, 9th pass, and 19th pass of the intruder. In each curve, the velocity indeed exhibits an overshoot behind the intruder resembling the flow in the case where the foam flows past an obstacle [23]. There, the intensity of the negative wake, while remaining constant in time, could be modulated using the obstacle height. Here, the maximum of the velocity overshoot clearly shows a time dependence decreasing as the number of intruder passes increases. The difference between the two systems occurs for obvious reasons: during the flowing foam experiment the foam is created on the fly and therefore is in a 'pristine' state when it hits the obstacle. This means that the internal stress field due to frustration is generated during the foam build-up. In the intruder case, the frustration in the foam changes due to the intruder at each pass. Interestingly enough, the negative wake does not decrease only at the first pass, but it continues to evolve even after 5 passes. The negative wake might decrease for instance due to the reorganization of the bubble configuration i.e. release of the local frustration due to geometry (residual stresses), the change 


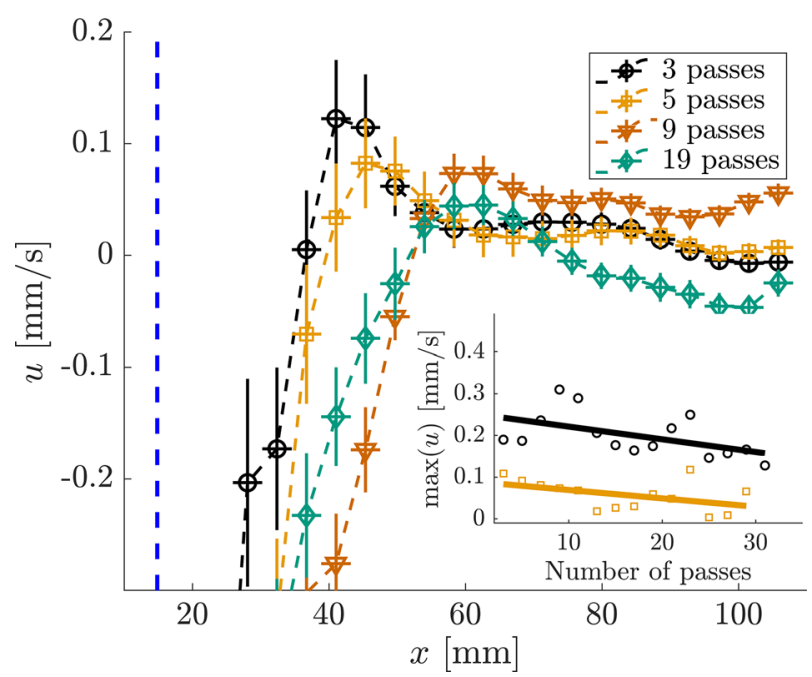

Fig. 5. The centerline profile of the velocity fields shown in Figure 4 with a single additional profile (9 intruder passes). The legend indicates the number of intruder passes before the time window over which the averaging is performed. The $x$-axis starts from the center of the obstacle and the vertical line labels the obstacle edge position. The orange curve in the inset shows the maximum velocity at the negative wake with respect to the number of intruder passes and guides to the eye. The black curve is a same experiment with much larger gas fraction.

in the average bubble size on the intruder trajectory due to the drifting of the bubbles caused by the intruder, or a change in the local volume fraction on the trajectory.

The inset of Figure 5 shows the peak value of velocity $u$-component in the negative wake for different intruder passes. In addition to the velocity evolution in the wet sample, the peak velocities for a dry foam sample are shown as well. However, the data is much noisier compared to the wet foam case, making the trend of decreasing overshoot less exposed. It is unclear, if this noise has a physical origin, possibly related to the higher yield stress of the dry foam, or if its due to the measurement setup. Also, for the current measuring protocol and the materials used, coarsening of the sample starts to be a problem in the dry foam case. Due to these issues, in what follows, we concentrate on the wet foam. There is some noise in the wet foam but it is partly a result from violent stick-slip motion of the intruder that is observed in videos.

Now we turn our attention to the relaxation mechanism. For this purpose, we identify the geometrical properties of the system, which change at each pass. Figure 6a shows an example of the Voronoi tessellation of the bubble configuration. As the plot shows, in the tessellation the fluid is included in the size of the bubbles, i.e. the bubble area can grow either due to the increase in the amount of fluid or the amount of gas. From the Voronoi tessellation we computed the number of nearest neighbors, which was independent of the number of passes at each bin. Therefore, we conclude that the change in the configuration is not due to the change in the bubble coordination. Then we are left with the options that either the volume fraction or bubble area would change locally due to the movement of the intruder. In Figure $6 \mathrm{~b}$ we plot the average bubble
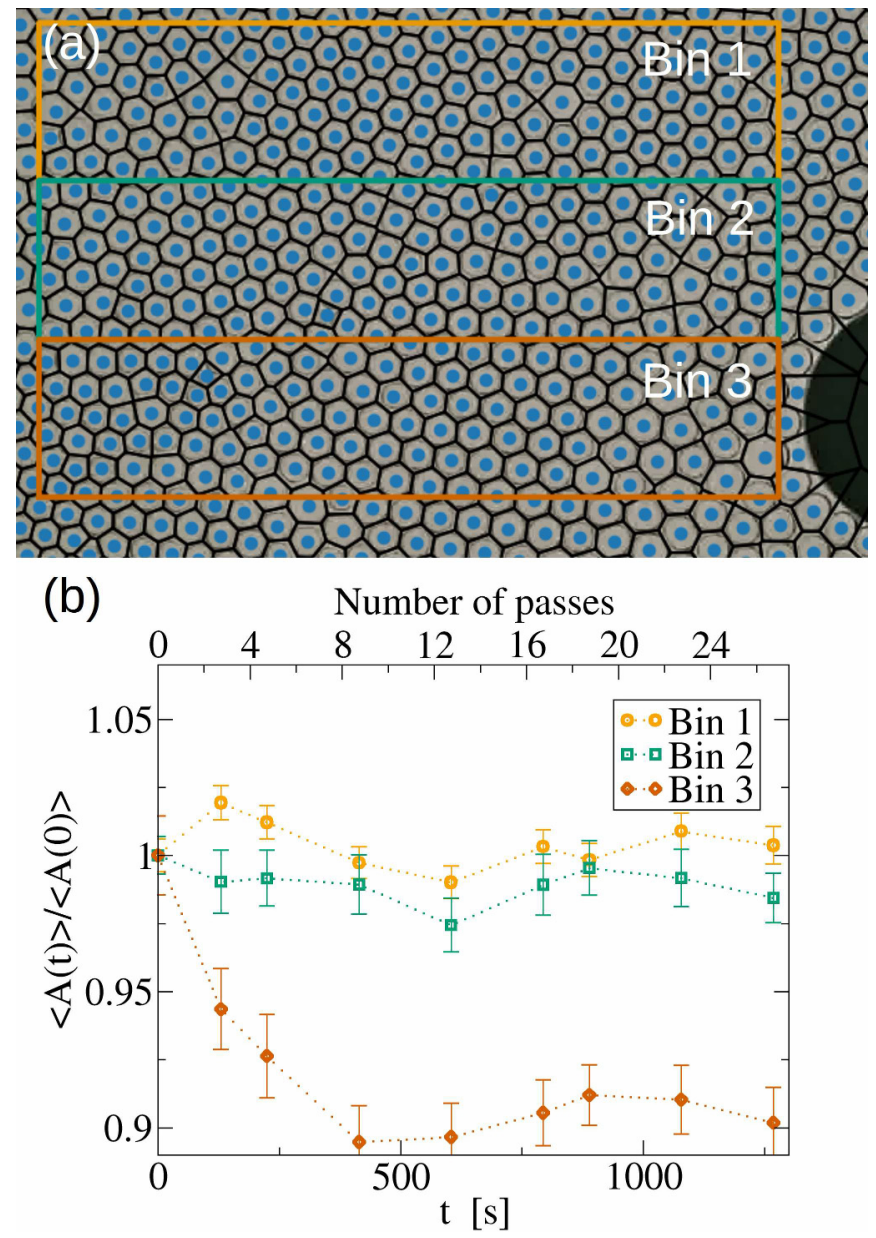

Fig. 6. (a) Voronoi tessellation based on bubble centers (blue dots). The area of each bubble is the area of the corresponding Voronoi cell. The mean bubble area is computed averaging over the shown bins. (b) In the area on the track of the intruder, the mean bubble area decreases with the number of passes (bin 3). Similar area decrease is not detected at the edges (bins 1 \& 2).

size behind the obstacle at three different regions, Bin 1, Bin 2, and Bin 3, as a function of time computed from the Voronoi tessellation of Figure 6a. Directly behind the intruder (Bin 3), the average bubble area drops in time, whereas the average area is independent of time in the other two bins. This means that in the intruder trail the number of bubbles increases with each pass. The bubbles are spherical within measurement accuracy due to low gas fraction. However, in a dryer case a structure factor $[31,32]$ could be calculated instead of the bubble area. If the volume fraction would decrease locally, the number of Voronoi cells would be decreasing, ruling out this possibility. Thus, this can be the result of large bubbles being substituted by smaller ones on the course of the intruder movement or due to the breakage of bubbles as the intruder hits them. The latter was not observed during the course of the experiment, as can be visually observed in the supplemental video. However, both Figures 5 and 6b (Bin 3) show the same trend, where the velocity and the structure both evolve during the first ten passes, after which they both saturate. 


\section{Conclusions}

In this article, we performed experiments with aqueous foam in a Hele-Shaw cell to study the flow field generated by a moving intruder. We observed the flow characteristics of the quadrupolar viscoelastic flow field, the negative wake, behind the intruder. In the setup, the intensity of the negative wake decreased with the number of intruder passes. We attributed this to the change in the bubble area computed from the Voronoi tessellation of the geometry. This could be related to the change in the gas volume or to a geometrical effect due to the transfer of large bubbles away from the obstacle trajectory. Voronoi tessellation did not allow us to distinguish between these two mechanisms, suggesting a more sophisticated analysis will be required for that. Determining the volume fraction from a quasi $2 \mathrm{D}$ system using $2 \mathrm{D}$ images even qualitatively is a formidable task, and doing it accurately (even a 1\% difference in gas fraction makes a huge difference here) is impossible. However, our result conforms perfectly with earlier observations on dry foams [25,26], where a moving intruder leaves a trace, a yielded region. When the intruder is followed by a second one, an attraction between the two intruders can be observed. Our experiment takes this a step further, and demonstrates that such trace in wet foams is rather permanent, and strengthens further as the number of intruder passes increases.

Future work should involve studying quantitatively the dependence of the velocity overshoot on the gas volume fraction, as well as the number of passes. This will require a more detailed control of the gas and suspension inlet flows. Likewise, it will be of interest to study driving the intruder at still lower velocities when clear stick-slip dynamics should be seen. Presently, it is unclear how the foam polydispersity changes the local velocity field around the intruder. It is well known that the polydispersity influences the critical jamming volume fraction, and therefore, changes the foam yield stress. Another interesting direction would be to perform direct comparisons between the present intruder system against the flowing foam past an obstacle case. This could help to understand the influence of boundary conditions due to the Hele-Shaw cell to the foam flow.

Open access funding provided by Aalto University. We acknowledge Academy of Finland through projects 278367, 308235, and support from Russian MegaGrant No. 14.W03.31.0002. Aalto Science IT project is acknowledged for computational resources. We also thank Christophe Raufaste for enlightening discussions.

\section{Author contribution statement}

L.V., J.K., A.P., and M.A. designed the measurement. L.V. and J.K. built the measurement device. L.V. performed the measurements and data analysis. All authors wrote and reviewed manuscript.

Open Access This is an open access article distributed under the terms of the Creative Commons Attribution
License (http://creativecommons.org/licenses/by/4.0), which permits unrestricted use, distribution, and reproduction in any medium, provided the original work is properly cited.

\section{References}

1. F. Bolton, D. Weaire, Phys. Rev. Lett. 65, 3449 (1990)

2. A. Saint-Jalmes, D. Durian, J. Rheol. 43, 1411 (1999)

3. G. Katgert, B.P. Tighe, M. van Hecke, Soft Matter 9, 9739 (2013)

4. K. Khakalo, K. Baumgarten, B.P. Tighe, A. Puisto, arXiv:1706.03932 (2017)

5. M. Van Hecke, J. Phys.: Condens. Matter 22, 033101 (2009)

6. S. Cohen-Addad, R. Höhler, O. Pitois, Annu. Rev. Fluid Mech. 45, 241 (2013)

7. K.A. Brakke, Exp. Math. 1, 141 (1992)

8. D.L. Weaire, S. Hutzler, The physics of foams (Oxford University Press, 2001)

9. R. Höhler, S. Cohen-Addad, J. Phys.: Condens. Matter 17, R1041 (2005)

10. J. Boschan, D. Vågberg, E. Somfai, B.P. Tighe, Soft Matter 12, 5450 (2016)

11. V. Bulatov, A. Argon, Model. Simul. Mater. Sci. Eng. 2, 167 (1994)

12. M.L. Falk, J.S. Langer, Phys. Rev. E 57, 7192 (1998)

13. A. Gopal, D. Durian, J. Colloid Interface Sci. 213, 169 (1999)

14. D. Weaire, Curr. Opin. Colloid Interface Sci. 13, 171 (2008)

15. H. Princen, in Encyclopedic Handbook of Emulsion Technology, edited by J. Sjöblom (Marcel Dekker Inc., 2001), p. 243

16. P. Marmottant, F. Graner, Soft Matter 9, 9602 (2013)

17. B. Dollet, F. Elias, C. Quilliet, A. Huillier, M. Aubouy, F. Graner, Colloids Surf. A 263, 101 (2005)

18. B. Dollet, F. Elias, C. Quilliet, C. Raufaste, M. Aubouy, F. Graner, Phys. Rev. E 71, 031403 (2005)

19. B. Dollet, F. Graner, J. Fluid Mech. 585, 181 (2007)

20. C. Raufaste, B. Dollet, S. Cox, Y. Jiang, F. Graner, Eur. Phys. J. E 23, 217 (2007)

21. B. Dollet, M. Durth, F. Graner, Phys. Rev. E 73, 061404 (2006)

22. O. Hassager, Nature 279, 402 (1979)

23. T. Chevalier, J. Koivisto, N. Shmakova, M. Alava, A. Puisto, C. Raufaste, S. Santucci, J. Phys.: Conf. Ser. 925, 012025 (2017)

24. I. Cheddadi, P. Saramito, B. Dollet, C. Raufaste, F. Graner, Eur. Phys. J. E 34, 1 (2011)

25. I.T. Davies, S.J. Cox, Colloids Surf. A 344, 8 (2009)

26. A. Wyn, I.T. Davies, S.J. Cox, Eur. Phys. J. E 26, 81 (2008)

27. A. Saint-Jalmes, Soft Matter 2, 836 (2006)

28. J.A. Glazier, S.P. Gross, J. Stavans, Phys. Rev. A 36, 306 (1987)

29. W. Thielicke, E. Stamhuis, J. Open Res. Softw. 2, 30 (2014)

30. C. Quilliet, S.A. Talebi, D. Rabaud, J. Käfer, S. Cox, F. Graner, Phil. Mag. Lett. 88, 651 (2008)

31. F. Moučka, I. Nezbeda, Phys. Rev. Lett. 94, 040601 (2005)

32. O. Ramos, E. Altshuler, K.J. Måløy, Phys. Rev. Lett. 102, $078701(2009)$ 